\title{
ОПТИМАЛЬНОЕ ПЛАНИРОВАНИЕ ОБЪЕМОВ СТРОИТЕЛЬНОГО ПРОИЗВОДСТВА В НЕСТАБИЛЬНОЙ ИНВЕСТИЦИОННОЙ СТРОИТЕЛЬНОЙ СРЕДЕ
}

\author{
(c) 2019 Айгумов Тимур Гаджиевич \\ кандидат экономических наук, доцент \\ заведующий кафедрой программного обеспечения вычислительной техники \\ и автоматизированных систем \\ Дагестанский государственный технический университет, Россия, Махачкала \\ E-mail:913355@mail.ru \\ (C) 2019 Мелехин Владимир Борисович \\ доктор технических наук \\ профессор кафедры программного обеспечения вычислительной техники \\ и автоматизированных систем \\ Дагестанский государственный технический университет, Россия, Махачкала \\ профессор кафедры прикладной математики и информационных технологий \\ Дагестанский государственный университет народного хозяйства, Россия, Махачкала \\ E-mail: pashka1602@rambler.ru
}

Рассмотрены основные проблемы, связанные с организацией управления и оптимального планирования объемов производства строительной компании в нестабильной инвестиционной строительной среде. В соответствии с наиболее часто встречающимися ситуациями динамичной инвестиционной строительной среды сформулированы соответствующие им задачи, решение которых позволяет строительной компании спланировать оптимальные объемы производства различных видов строительной продукции, производимой хозяйственным способом и на этой основе получить максимально возможную прибыль. Предложены оригинальные методики решения поставленных оптимизационных задач позволяющие сформировать соответствующие им информационные технологии и на этой основе автоматизировать процесс планирования производственной деятельности строительной компании.

Ключевые слова: строительная компания, оптимальные объемы производства, нестабильная среда, максимальная прибыль, риски.

Введение. Одной из актуальных проблем экономики строительного производства является оптимальное планирование объемов различного вида готовой строительной продукции в нестабильной инвестиционной строительной среде (ИСС). Особенно остро данная проблема стоит перед строительными компаниями (СК), занимающимися строительством различных объектов хозяйственным способом. В общем случае, при хозяйственном способе строительства, как правило, объектов жилищного и гражданского назначения к основным инструментам эффективного управления производственной деятельностью СК следует отнести.

1. Планирование оптимальных объемов производства исходя из:

- мощности производственного потенциала;
- объемов неудовлетворенного спроса на различные виды строительной продукции на различных сегментах рынка;

- прироста (снижения) объемов производства с учетом тенденций изменения спроса на производимые виды строительной продукции.

2. Регулирование производственных процессов на основе:

- эффективной организации строительного производства с учетом имеющейся у СК строительной техники и средств механизации, например, путем оптимального перемещения строительной техники по строящимся объектам;

- управлению поставками и запасами строительных материалов и конструкций согласно заданному опережению графиков поставок потребностей производственного процесса в строительных материалах с целью бесперебойной 
реализации запланированных объемов производства;

- корректировки производственных планов с учетом непредвиденных изменений, происходящих в ИСС.

3. Сбалансированное управление стоимостью и качеством производимой строительной продукции на основе:

- корректировки производственных технологий с учетом качества имеющихся на рынке строительных материалов и конструкций, учитывая покупательскую способность потребителей на различных сегментах рынка;

- организации эффективного входного контроля качества поступающих от поставщиков строительных материалов и конструкций;

- промежуточного и выходного контроля над соблюдением технологий строительства различных циклов строительно-монтажных работ;

- корректировки производственных технологий с учетом состояния, структуры и качества используемых в производственном процессе строительных материалов и конструкций для получения запланированного уровня качества строительной продукции на выходе;

- сбалансирования издержек и качества производимой строительной продукции с учетом покупательской способности основной массы потребителей при ее свободной реализации на различных сегментах рынка.

Следует отметить, что в научной литературе достаточно полное отражение нашли принципы организации, инструментальные средства и методы, обеспечивающие эффективное решение большинства отмеченных выше задач управления производственным процессом в строительстве и качеством производимой продукции. Например, эффективной организации строительного производства посвящены работы [7, 9], решение основных проблем связанных с управлением поставок и управлением запасами рассматривается в [5, 8], принципы управления качеством строительной продукции излагаются в работах $[3,5]$ и т.д. Однако проблеме оптимального планирования объемов строительного производства в изменяющихся условиях ИСС не уделяется должного внимания. Хотя без эффективного решения данной проблемы, т.е. без разработки эффективных инструментальных средств цифрового регулирования объемов производства, невозможно создать информацион- ные технологии, позволяющие обеспечить принятие эффективных управленческих решений на основе цифровых данных в нестабильных условиях современной ИСС, а также снизить издержки, связанные с производством хозяйственным способом различных видов строительной продукции. В этой связи, возникает объективная необходимость в проведении более глубоких исследований основных проблем, связанных с разработкой организационно-экономических основ и инструментальных средств оптимального планирования объемов строительного производства в нестабильных условиях функционирования.

Постановка задач и методики оптимального планирования объемов строительного производства. Учитывая наиболее часто встречающиеся ситуации в инвестиционной строительной среде, к основным задачам оптимального планирования объемов строительного производства следует отнести:

- планирование производства таких объемов внедряемой в производство готовой строительной продукции, которые позволяют обеспечить получение максимально возможной прибыли и удовлетворить требования потребителей на различных сегментах рынка с учетом спроса на эту продукцию;

- планирование таких объемов различного вида строительной продукции пользующейся на рынке спросом, которые обеспечивают получение максимально возможной прибыли с учетом ограничений на имеющиеся у СК инвестиционные ресурсы и риски, обусловленные снижением спроса на различные виды строительной продукции в течение отчетного периода $\mathrm{T}_{\mathrm{O}}$;

- планирование оптимального прироста объемов производства, которые сбалансированы с изменяющимся спросом на производимую СК продукцию, с учетом имеющихся инвестиционных средств с целью получения дополнительной прибыли.

Для построения информационных технологий, обеспечивающих СК возможность автоматизированного планирования оптимальных объемов производства в рассмотренных выше ситуациях ИСС формализуем постановку и рассмотрим технологии решения следующих оптимизационных задач.

3 а д а ч а 1. Пусть у СК имеются ограниченные инвестиционные ресурсы Z, которые она планирует использовать для роста объемов про- 
изводства путем внедрения новых видов строительной продукции. В основном данные средства при хозяйственном способе строительства расходуются на приобретение материальных ресурсов требуемого качества, которые необходимы для производства различных $\mathrm{j}(\mathrm{j}=1,2, .$. , $\mathrm{m}^{*}$ ) видов строительной продукции в объемах не более объемов $V_{j}^{*}$ неудовлетворенного на нее спроса. Необходимо так распределить инвестиционные ресурсы Z между различными видами внедряемой в производство строительной продукции, чтобы обеспечить минимальные суммарные издержки производства и максимизировать на этой основе получаемую СК суммарную прибыль Р, где $\mathrm{m}^{*}$ - общее количество различных видов строительных объектов, которые способно производить СК.

Уточним необходимые для решения поставленной задачи обозначения и исходные данные: $\mathrm{d}_{\mathrm{i}}(\mathrm{j}), \mathrm{i}=1,2, \ldots, \mathrm{m}_{\mathrm{j}}$ - различные виды материальных ресурсов, необходимые для строительства одного объекта ј-го вида удовлетворяющего требованиям потребителей на выбранном сегменте рынка, где $\mathrm{m}_{\mathrm{j}}$ - общее количество различных видов материальных ресурсов; $\mathrm{V}_{\mathrm{i}}(\mathrm{j})$, $\mathrm{j}=1, \mathrm{~m}^{*}$ - объемы і вида материальных ресурсов, требующиеся для производства одной единицы j вида строительной продукции; $\mathrm{t}_{\mathrm{k}}^{*}(\mathrm{j}), \mathrm{k}=1,2, \ldots, \mathrm{n}_{\mathrm{j}}$ - различные виды требований потребителей к j виду строительной продукции, где $\mathrm{n}_{\mathrm{j}}$ - общее количество требований предъявляемых потребителями к j виду объектов; $\Delta \mathrm{C}_{\mathrm{ik}}(\mathrm{j})-$ стоимость единичного объема і вида строительных материалов, обладающих требуемым качеством, позволяющим удовлетворить k-e требование потребителей к ј виду строительных объектов; $\Delta \mathrm{S}_{\mathrm{j}}$ - издержки, связанные с производством и реализацией одной единицы строительной продукции $\mathrm{j}$ вида; $\mathrm{C}_{\mathrm{j}}$ - ориентировочная рыночная стоимость одной единицы строительной продукции ј вида на заданном сегменте рынка строительной продукции.

Используя принятые выше обозначения сформулированную ранее оптимизационную задачу в формализованном виде можно представить следующим образом. С учетом ограничений на имеющиеся инвестиционные ресурсы $\mathrm{Z}$, найти такие объемы производства $\mathrm{V}_{\mathrm{j}}$ или количество $\mathrm{n}_{\mathrm{j}}$ внедряемых в производство объектов каждого ј вида, удовлетворяющих требованиям потребителей $\mathrm{t}_{\mathrm{k}}^{*}(\mathrm{j}), \mathrm{k}=1,2, \ldots, \mathrm{n}_{\mathrm{j}}$ при которых получаемая СК суммарная прибыль $\mathrm{P}$, принима- ет максимальное значение:

$$
\mathrm{P}=\sum_{\mathrm{j}=1}^{\mathrm{m}^{*}} \mathrm{~V}_{\mathrm{j}}\left(\mathrm{C}_{\mathrm{j}}-\sum_{\mathrm{i}=1}^{\mathrm{m}_{\mathrm{j}}} \sum_{\mathrm{k}-1}^{\mathrm{n}_{\mathrm{j}}} \mathrm{V}_{\mathrm{i}}(\mathrm{j}) \Delta \mathrm{C}_{\mathrm{ik}}(\mathrm{j})\right) \Rightarrow \max
$$

при следующих ограничениях:

$$
\left(\sum_{\mathrm{j}=1}^{\mathrm{m}^{*}} \sum_{\mathrm{i}=1}^{\mathrm{m}_{\mathrm{j}}} \sum_{\mathrm{k}=1}^{\mathrm{n}_{\mathrm{j}}} \mathrm{V}_{\mathrm{j}} \mathrm{V}_{\mathrm{i}}(\mathrm{j}) \mathrm{C}_{\mathrm{ik}}(\mathrm{j})\right) \leq Z ; 0 \leq \mathrm{V}_{\mathrm{j}} \leq \mathrm{V}_{\mathrm{j}}^{*}
$$

Таким образом, имеет место задача условной оптимизации [7], для решения которой предлагается следующая методика. (Другими словами решение данной задачи определяется на границах допустимых значений объемов производства $V_{j}^{*}$ внедряемой в производственный процесс строительной продукции).

Начало. 1. Ранжировать различные виды строительной продукции слева направо в порядке убывания получаемой прибыли $\mathrm{V}_{\mathrm{j}}^{*}\left(\mathrm{C}_{\mathrm{j}}-\Delta \mathrm{S}_{\mathrm{j}}\right)$ от реализации ее допустимых объемов, равных неудовлетворенному на нее спросу $\mathrm{V}_{\mathrm{j}}^{*}$.

2. Выбрать в полученном кортеже первый вид продукции. Определить инвестиции $\mathrm{Z}_{1}$ требующиеся для производства первого вида продукции в объемах равных $\mathrm{V}_{1}^{*}$ :

$$
\mathrm{Z}_{1}=\mathrm{V}_{1}^{*} \sum_{\mathrm{i}=1}^{\mathrm{m}_{1}} \sum_{\mathrm{k}=1}^{\mathrm{n}_{1}} \mathrm{~V}_{\mathrm{i}}(1) \mathrm{C}_{\mathrm{ik}}(1)
$$

3. Проверить условие « $\left(\mathrm{Z}-\mathrm{Z}_{1}\right)>0 »$ : если условие выполняется, то перейти к п. 4; в противном случае, перейти к п. 5.

4. Определить объемы $\mathrm{V}_{1}$ первого вида продукции, которые можно произвести, используя имеющиеся инвестиции в объеме равном Z:

$$
\mathrm{V}_{1}=\frac{\mathrm{Z}}{\Delta \mathrm{S}_{1}}
$$

где $\Delta \mathrm{S}_{1}$ - издержки производства и реализации одного объекта первого вида, перейти к п. 6.

5. Вычислить оставшиеся инвестиционные средства: $Z_{0}^{1}=Z-Z_{1}$. Выбрать в полученном кортеже второй вид продукции. Проверить условие «инвестиции в объеме $Z_{0}^{1}$ позволяют произвести второй вид продукции в объемах, равных ее неудовлетворенному спросу $\mathrm{V}_{2}^{*}$, т.е.

$$
\frac{\mathrm{Z}_{\mathrm{O}}^{1}}{\Delta \mathrm{S}_{2}} \geq \mathrm{V}_{2}^{*} »:
$$

если условие выполняется, то определить объемы инвестиций $Z_{2}$ необходимые для производства второго вида строительной продукции в 
объемах, равных $\mathrm{V}_{2}^{*}$ :

$$
\mathrm{Z}_{2}=\mathrm{V}_{2}^{*} \sum_{\mathrm{i}=1}^{\mathrm{m}_{1}} \sum_{\mathrm{k}=1}^{\mathrm{n}_{1}} \mathrm{~V}_{\mathrm{i}}(2) \mathrm{C}_{\mathrm{ik}}(2)
$$

Оставшиеся инвестиции $\mathrm{Z}_{0}^{2}=\mathrm{Z}_{0}^{1}-\mathrm{Z}_{2}$ перераспределить по вышеописанному принципу между стальными видами продукции с учетом получаемой на их основе прибыли, перейти к п. 6; в противном случае, определить объемы производства $\mathrm{V}_{2}$ второго вида строительной продукции исходя из оставшихся инвестиций $\mathrm{Z}_{0}^{1}$,

$$
\mathrm{V}_{2}=\frac{\mathrm{Z}_{\mathrm{o}}^{1}}{\Delta \mathrm{S}_{2}} \text {, перейти к п. } 6 .
$$

6. Инвестиции распределены. Конец.

3 а д а ч а 2. Допустим, что в экономической среде СК вполне вероятно может возникнуть проблемная ситуация, при которой наблюдается тенденция снижения объемов спроса на планируемые к внедрению в производство виды строительной продукции. Данная ситуация, как правило, на практике возникает в результате повышения цен на различные виды строительных материалов, которое в течение определенного периода времени может привести к такому росту цен на готовую строительную продукцию, в результате которого они будут превышать покупательскую способность основной массы потребителей на соответствующем сегменте рынка.

Пусть для определения рисков связанных со снижением спроса на различные виды строительной продукции в СК используется методика, предложенная в [1]. Тогда, если по истечению отрезка времени $\Delta \mathrm{T}$ наблюдаются риски снижения спроса для каждого ј вида строительной продукции, равные $\gamma_{j}$, то скорость $\Delta \gamma_{j}$ нарастания данных рисков в единицу времени можно вычислить следующим образом:

$$
\Delta \gamma_{\mathrm{j}}=\frac{\gamma_{\mathrm{j}}}{\Delta \mathrm{T}}
$$

Тогда, если провести ранжирование различных видов строительной продукции в порядке убывания получаемой прибыли с учетом скорости нарастания рисков $\Delta \gamma_{j} V_{j}^{*}\left(C_{j}-\Delta S_{j}\right)$ с учетом допустимых объемов ее производства $V_{j}^{*}$ в текущий момент времени, то на основе приведенной выше методики можно определить оптимальные объемы производства различного вида строительной продукции, удовлетворяющие критерию получения максимально возмож- ной прибыли. Кроме того, полученные объемы производства различных видов строительной продукции будут найдены с учетом вероятности проявления в экономической среде рисковых событий и степени их влияния на производственный процесс [1].

При высоких вероятностях возникновения рисковых событий задачу оптимального планирования объемов производства различного вида строительной продукции целесообразно сформулировать в следующем виде.

Найти такие объемы производства $\mathrm{V}_{\mathrm{j}}$ или количество $\mathrm{n}_{\mathrm{j}}$ внедряемых в производство строительных объектов каждого ј вида, удовлетворяющих требованиям потребителей $\mathrm{t}_{\mathrm{k}}^{*}(\mathrm{j}), \mathrm{k}=1,2, \ldots, \mathrm{n}_{\mathrm{j}}$ и при которых получаемая СК суммарная прибыль Р, принимает максимальное значение:

$$
\mathrm{P}=\sum_{\mathrm{j}=1}^{\mathrm{m}^{*}} \Delta \gamma_{\mathrm{j}} \mathrm{V}_{\mathrm{j}}\left(\mathrm{C}_{\mathrm{j}}-\sum_{\mathrm{i}=1}^{\mathrm{m}_{\mathrm{j}}} \sum_{\mathrm{k}-1}^{\mathrm{n}_{\mathrm{j}}} \mathrm{V}_{\mathrm{i}}(\mathrm{j}) \Delta \mathrm{C}_{\mathrm{ik}}(\mathrm{j})\right) \Rightarrow \max
$$

а суммарная скорость нарастания рисков $\Delta \gamma$ минимизируются:

$$
\Delta \gamma=\sum_{\mathrm{j}=1}^{\mathrm{m}^{*}} \Delta \gamma_{\mathrm{j}} \Rightarrow \min
$$

при следующих ограничениях

$$
\left(\sum_{\mathrm{j}=1}^{\mathrm{m}^{*}} \sum_{\mathrm{i}=1}^{\mathrm{m}_{\mathrm{j}}} \sum_{\mathrm{k}=1}^{\mathrm{n}_{\mathrm{j}}} \Delta \gamma_{\mathrm{j}} \mathrm{V}_{\mathrm{j}} \mathrm{V}_{\mathrm{i}}(\mathrm{j}) \mathrm{C}_{\mathrm{ik}}(\mathrm{j})\right) \leq Z ; 0 \leq \mathrm{V}_{\mathrm{j}} \leq \mathrm{V}_{\mathrm{j}}^{*}
$$

Таким образом, имеет место задача двухкритериальной оптимизации, решение которой методом Парето [6] позволяет определить компромиссно оптимальные объемы $\mathrm{V}_{j}^{* *} \leq \mathrm{V}_{j}^{*}$ внедряемой в производство строительной продукции при условно минимальных значениях рисков, возникающих в нестабильной инвестиционной строительной среде СК.

3 а д а ч а 3. Пусть в экономической среде наблюдается рост покупательской способности потребителей на производимую СК продукцию. Тогда СК может поступить следующим образом:

- если отсутствуют инвестиции необходимые для дальнейшего развития, то СК может повышать стоимость реализуемой готовой продукции пропорционально объемам роста неудовлетворенного на нее спроса, выполнив, таким образом, сбалансирование спроса и предложения на рынке на соответствующие виды строительной продукции;

- при наличии инвестиционных средств $\mathrm{Z}^{\text {** }}$ 
позволяющих увеличить объемы производимой продукции с целью получения дополнительной прибыли, превышающей прибыль $\Delta \mathrm{P}_{\mathrm{j}}$, получаемую СК за счет роста цен на производимую продукцию ј вида, ей целесообразно выполнить прирост объемов производства.

Следует отметить, что СК целесообразно выполнить прирост $\Delta \mathrm{V}_{\mathbf{j}}$ объемов производства j вида рентабельной продукции, если выполняется условие:

$$
\Delta \mathrm{V}_{\mathrm{j}}>\frac{\Delta \mathrm{P}_{\mathrm{j}}}{\mathrm{C}_{\mathrm{j}}-\Delta \mathrm{S}_{\mathrm{j}}}-\mathrm{V}_{\mathrm{j}}^{*}
$$

при справедливости следующего неравенства:

$$
\left(\sum_{\mathrm{j}=1}^{\mathrm{m}^{*}} \sum_{\mathrm{i}=1}^{\mathrm{m}_{\mathrm{j}}} \sum_{\mathrm{k}=1}^{\mathrm{n}_{\mathrm{j}}} \Delta \mathrm{V}_{\mathrm{j}} \mathrm{V}_{\mathrm{i}}(\mathrm{j}) \mathrm{C}_{\mathrm{ik}}(\mathrm{j})\right) \leq \mathrm{Z}_{\mathrm{j}}^{* *}
$$

где $\mathrm{Z}_{\mathrm{j}}^{\text {*** }}$ - имеющиеся у СК инвестиционные средства на момент времени рассмотрения вопроса о целесообразности увеличения объемов производства ј вида строительной продукции.

Необходимо отметить, что соотношение (1) получено в результате преобразования следующего неравенства: $\left(\mathrm{V}_{\mathrm{j}}+\Delta \mathrm{V}_{\mathrm{j}}\right)\left(\mathrm{C}_{\mathrm{j}}-\Delta \mathrm{S}_{\mathrm{j}}\right)>\Delta \mathrm{P}_{\mathrm{j}}$, определяющего условие, при выполнении которого СК за счет роста объемов производства на величину равную $\Delta \mathrm{V}_{\mathrm{j}}$, может получить дополнительную прибыль превышающую прибыль, которую она будет иметь за счет допустимого роста цен на ј вид строительной продукции.

В результате решения задач (1-3) в зависимости от сложившейся в ИСС ситуации, СК планирует такие объемы $\mathrm{V}_{1}, \mathrm{~V}_{2}, \ldots, \mathrm{V}_{\mathbf{j}}$ производства каждого вида наиболее рентабельной строительной продукции и на этой основе формирует производственную программу, которая позволяет ей получить максимально возможную прибыль с учетом имеющихся у нее инвестиционных средств на развитие.

Следует также отметить, что все рассмотренные выше задачи решались без учета ограничений на потенциальные возможности СК. Однако любая СК имеет ограниченные потенциальные возможности относительно объемов производства различного вида строительных работ. В этой связи после определения оптимальных объемов производства $\mathrm{V}_{1}, \mathrm{~V}_{2}, \ldots, \mathrm{V}_{\mathrm{j}}$ различного вида строительной продукции возникает необходимость в их ограничении с учетом потенциальных возможностей СК. Для этого необходимо решить следующую оптимизационную задачу.
3 а д а ч а 4. Ограничить найденные оптимальные объемы производства $V_{i_{2}}: n_{i_{2}}, i_{2}=1,2, \ldots j$, различных видов рентабельной строительной продукции с учетом суммарных объемов $\mathrm{V}_{\beta}^{*}$, $\beta=1,2, \ldots, \mathrm{m}_{\beta}$ различного вида строительных работ, которые СК имеет возможность выполнить согласно своим потенциальным возможностям, при условии обеспечения максимума $\mathrm{P}^{*}$ получаемой прибыли. В формальном виде данная задача определяется следующим образом. Найти такое количество $n_{i_{2}}$ каждого вида объектов при ограниченных объемах $\mathrm{V}_{\beta}^{*}, \beta=1,2, \ldots, \mathrm{m}_{\beta}$ выполняемых строительных работ, для которых выполняется следующее условие:

$$
\mathrm{P}^{*}=\sum_{\mathrm{i}_{2}}^{\mathrm{j}} \sum_{\beta=1}^{\mathrm{m}_{\beta}} \mathrm{n}_{\mathrm{i}_{2}} \mathrm{~V}_{\beta}\left(\mathrm{C}_{\mathrm{i}_{2}}-\Delta \mathrm{S}_{\mathrm{i}_{2}}\right) \Rightarrow \max
$$

при ограничениях вида:

$$
\sum_{\mathrm{i}_{2}-1}^{\mathrm{j}} \mathrm{n}_{\mathrm{i}_{2}} \mathrm{~V}_{\beta} \leq \mathrm{V}_{\beta}^{*}, \beta=1,2, \ldots, \mathrm{m}_{\beta}, n_{i_{2}} \geq 0 .
$$

В приведенной выше задаче использованы следующие обозначения: $\mathrm{V}_{\beta}\left(\mathrm{i}_{2}\right)$ - объемы работ вида $\beta$, выполняемые в процессе строительства одного объекта $\mathrm{i}_{2}$ типа; $\mathrm{C}_{\mathrm{i}_{2}}, \Delta \mathrm{S}_{\mathrm{i}_{2}}$ - соответственно рыночная стоимость и издержки производства, а также реализации одного объекта $\mathrm{i}_{2}$ типа. Для решения данной задачи целесообразно использовать метод линейного программирования [2].

Необходимо отметить, что двухэтапный расчет оптимальных объемов производства различного вида строительной продукции, например, путем решения задач (1) и (4), используется для сокращения пространства поиска их оптимального решения.

Заключение. Резюмируя вышеизложенное, можно сделать следующие основные выводы.

1. Сформулированные в работе оптимизационные задачи позволяют СК планировать оптимальные объемы производства различных видов строительной продукции и строить на этой основе эффективные производственные программы. Решение данных задач с учетом имеющихся инвестиционных ресурсов, а также рисков, связанных с падением спроса на внедряемую в производственный процесс продукцию, обеспечивает СК эффективную производственную деятельность в нестабильной ИСС за счет получения максимально возможной прибыли.

2. Учитывая вычислительную сложность 
предложенных методов решения поставленных задач их целесообразно реализовать в виде информационных технологий, обеспечивающих возможность автоматизированного планирования оптимальных объемов производства в нестабильных условиях отечественного рынка.

3. Сформулированные оптимизационные задачи и методы их решения без принципиальных изменений могут быть использованы для планирования оптимальных объемов производства на производственных предприятиях, относящихся к различным отраслям национальной экономики.

\section{Библиографический список}

1. Абдурахманов Д.Б., Дуллуева Р.М., Мелехин В.Б. Методика интегральной оценки инвестиционных рисков строительного предприятия с нечеткой логикой обработки экспертных данных // Экономика строительства. 2014. № 4. С. 34-39.

2. Агальцов В.П. Математические методы в программировании. М.: Форум, 2015. 240 с.

3. Брячихин А.М. Управление качеством продукции строительства. М.: Стройиздат, 1989. 229 с.

4. Версин В.Г. Интеграция управления качеством продукции: Новые возможности. М.: Из-во стандартов, 1994. 218 c.

5. Линдер P.М., Фирон Е. Ч. Управление снабжением и запасами. Логистика / Пер. с англ. СПб.: Полигон, 2002. 768 c.

6. Растригин Л.А. Современные принципы управления сложными объектами. М.: Сов. Радио, 1980. 232 с.

7. Соколов Г.К. Технология и организация строительства. М.: Академия, 2018. 124 с.

8. Спектор В.А., Шор С. М., Куликова Л. С. Материально-техническое снабжение строительства: Справочник в 2-х. М.: Стройиздат, 1990. Т1-284 с., Т2-281 с.

9. Ширшиков Б. Ф. Организация, планирование и управление строительством. М.: АСВ, 2012. 528 с. 